\title{
IIoT Based Efficiency Optimization in Logistics Applications
}

Stefano Farné, Francesco Benzi \& Ezio Bassi

Department of Electrical, Computer and Biomedical Engineering, University of Pavia, Italy.

DOI: http://doi.org/10.38177/AJBSR.2020.2406

Copyright: @2020 Stefano Farné et al. This is an open access article distributed under the terms of the Creative Commons Attribution License, which permits unrestricted use, distribution, and reproduction in any medium, provided the original author and source are credited.

\section{ABSTRACT}

The Industrial Internet of Thing (IIoT) approach to an Industry plant design, devises a comprehensive interconnection of the system components, from sections up to single devices, in order to get a general and punctual understanding of the process. Such an intelligent network, mostly based on Ethernet basic layers, when properly conceived, should be able to add relevant value to the plant operation. This paper shows how, within the IIoT frame topics, the plant efficiency can be addressed and bring relevant improvement. The reason is that variables directly related to the energy consumption, such as current, electric power, actuator and motor torque, speed, etc., can be timely and easily monitored in the entire plant, since they are already conveyed on the network, due to real time control and diagnostics purpose. A power consumption diagram can be derived, and give hints on how to optimize operations, based on some efficiency index. The paper, after a general discussion, proves it with practical examples based on a Gantry robot, driven in an EtherCAT based automation network, and on the stacker cranes of an automated warehouse.

\section{Introduction}

The IoT (Internet of Things) expression describes a way along the technology development process based on the internet network, where each object available in daily use can be interconnected. IoT refers to smart machines with pervasive sensing, networked to the Cloud, and is distinct from M2M communication. IoT can be considered as an extension of the traditional wireless sensor networks (WSN) that makes the object-to-object communication possible by using Radio frequency identification (RFID) technology. It deals with connectivity in general purpose networks, warehousing infrastructure and building systems to enable the delivery of software services, data analytics and autonomous control from the Cloud. IIoT (Industrial Internet of Things) add the Industrial frame and constraints to the IoT, thus stressing some specific requirements, typical of Industry Control, such as Determinism, Real Time capability, Robustness and Reliability, Security and Integrity. In IIoT each object, be a physical device or an abstract entity, acquires a unique digital identity. Currently there are significant changes in the industrial process control, under the idea to reduce cost and improve efficiency by integrating the latest advances in software and communication techniques, therefore bringing cabled and wireless communication protocols and internet architectures as complementary tools [1-3].

The logistics chain for example is traditionally a complex problem, due to the difficulty for users to characterize services chain performance quantitatively: indeed different criteria may have different roles in various jobs [4-7]. On the other hand, when using IIoT tools, even logistics problems can be approached in a systematic way. The IoT system architecture is generally divided into three layers: the communication (or perception) layer, the network layer, the service layer (or application layer).

The communication layer is the information source and the core layer of IoT. All kinds of information of the physical world used in IoT are perceived and collected in this layer (e.g. wireless sensors network (WSN), tags and reader-writers etc.). Network layer, also called transport layer, includes access network and core network, and 
provides transparent data transmission capability. The information from perception layer can be sent to the upper layer using existing mobile communication network. Service layer, also called application layer, includes data management sub-layer and application service sub-layer. The data management sub-layer provides processing complex data and uncertain information.

Most of the "philosophy" implied by IoT and namely IIoT converges in the German originated project called Industry 4.0. The application of Internet of Things to Intelligent Warehouse Information Management System is an expanding field. Warehouse inventory management refers to the need to manage a large variety of items and the flow of materials in a timely manner. This management can take advantage by the information derived from the IoT and can lead to faster decisions that have positive influence on production planning, transport scheduling and, in general, on the supply chain efficiency [8-10]. Besides, Cloud manufacturing (CM) and IIoT are interlinked and can find a simultaneous application in the production logistics (PL) processes. CM and IIoT infrastructures can be systematically integrated to enable a control mechanism (such as smart PLS) with multi-level dynamic adaptability [11].

The paper will resume in paragraph II-IV the frame where industry applications can be viewed and improved in this context. Paragraph V and VI will illustrate this with examples based on an actual implementation of a Gantry architecture, Pick-and-Place robot and of the stacker cranes of an automated warehouse. Macros based on the EtherCAT protocol are also described to suggest an Energy efficiency improvement strategy.

\section{Industry 4.0 and Enabling Technologies for IIoT}

\section{A. Design Principles in Industry 4.0}

The term "Industry 4.0" originates from a project in the high-tech strategy of the German government, aiming at promoting the computerization of manufacturing [12]. The first industrial revolution mobilized the mechanization of production using water and steam power. The second industrial revolution then introduced mass production with the help of electric power and oil, followed by the digital revolution and the use of electronics and IT to further automate production.

There are six design principles in Industry 4.0. These principles support companies in identifying and implementing Industry 4.0 scenarios [13].

1. Interoperability: the ability of Cyber-Physical Systems, CPS (i.e. work piece carriers, assembly stations and products), humans and Smart Factories to connect and communicate with each other through open nets and semantic descriptions such as Internet of Things and the Internet of Services, thanks to the communication standards.

2. Virtualization: a virtual copy of the Smart Factory which is created by linking sensor data (from monitoring physical processes) with virtual plant models and simulation models. Virtualization means that CPS are able to monitor physical processes. These sensor data are linked to virtual plant models and simulation models. In case of failure, a human can be notified. In addition, all necessary information, like next working steps or safety arrangements, are provided. 
3. Decentralization: the ability of CPS within Smart Factories to make decisions on their own. Embedded computers enable CPS to make decisions on their own. Only in cases of failure, tasks are delegated to a higher level. The central controlling is no longer needed.

4. Real-Time Capability: the capability to collect and analyze data and provide the derived insights immediately. For organizational tasks, it is necessary that data be collected and analyzed in real time. The status of the plant is permanently tracked and analyzed. Thus, the plant can react to the failure of a machine and re-route products to another machine.

5. Service Orientation: offering of services (of cyber-physical systems, humans or Smart Factories) via the Internet of Services. Other participants can utilize the services inside the company. The company in turn exploits the network by sharing its facilities as a web service.

6. Modularity: flexible adaptation of Smart Factories to changing requirements by replacing or expanding individual modules. Based on standardized software and hardware interfaces new modules are identified automatically and can be immediately integrated and used.

On this basis, it is quite easy to point out an overlap not to say a coincidence between Industry 4.0 and IloT.

\section{B. Industrial Ethernet EtherCAT}

A possibility to implement the Industry 4.0 paradigm is based on the use of drives (PLC controllers, inverters...) connected by an intelligent bus system such as Industrial Ethernet (IE). Originally developed for telecommunications, the standard Ethernet is also used for industrial application such as machines and process control. Components used in plant process areas must be designed to work in harsh environments of extremes temperature, humidity, and vibration that exceeds the ranges for information technology equipment intended for installation in controlled environments. Plant-floor equipment must tolerate a wider range of temperature, vibration, and electrical noise than equipment installed in dedicated information-technology areas. This solution is called Industrial Ethernet, and makes reference to the application of the standard Ethernet protocol layers, for automation or process control, thus requiring more reliable behavior both in hardware and software.

Industrial Ethernet presents a strong feature for different operating needs. At the hardware level, due to the working environment within which it must operate, it is subject to thermal and vibrational stresses and this implies more marked coating solutions. In fact, it has coating shells and cooling systems. In terms of control and communication, even if both support IP standards, Industrial Ethernet communicates in an internal line and with a multi-cast system, unlike Ethernet that communicates outdoors and with a unicast system. Industrial networks use network switches to segment a large system into logical sub-networks, divided by address, protocol, or application. Using network switches allows the network to be broken up into many small collision domains. This reduces the risk of a faulty or mis-configured device generating excess network traffic.The switched topology therefore help making communications lines freer and more suited to manage data traffic by means of three different applications: Packet loss under congestion, broadcast and multicast, network analyzers. The first assigns a priority criterion to the information in circle so that it always and continuously arrives at the destination. The latter allows directing the 
information exclusively to the nodes involved in order to avoid a clogging of the network. And finally, the network analyzers allows real time diagnostics of the network, to avoid the most common types of attack and monitor input and output of individual nodes.

Since closed-loop process control may rely on an Ethernet link, economic cost of interruptions may be high and availability is, therefore, an essential criterion. So, Industrial Ethernet networks must provide predictable performance and maintainability. In addition to physical compatibility and low-level transport protocols, a practical industrial Ethernet system must also provide interoperability of higher levels of the OSI model. An industrial network must provide security both from intrusions from outside the plant, and from inadvertent or unauthorized use within the plant [14].

When an industrial network must connect to an office network or external networks, a firewall system is inserted to control exchange of data between the networks. To preserve the performance and reliability of the industrial network, general office automation systems are separated from the network used for control or I/O devices. PLC (Programmable logic controller) communicate using one of several possible open or proprietary protocols, such as EtherNet/IP, Modbus, Sinec H1, Profibus, CANopen, DeviceNet or FOUNDATION Fieldbus. The idea to use standard Ethernet makes these systems more inter-operable [15].

In the examples presented in paragraph III and IV it has been used EtherCAT - Ethernet for Control Automation Technology - that is an Ethernet-based fieldbus system, invented by Beckhoff Automation.

EtherCAT is a network used for complete and real-time control of servo drives, inverters, vision systems, I / O, robots, and security. It has the ability to control 192 slaves and a data transmission rate of $100 \mathrm{Mbps}$ under its control. Despite the use of an independent protocol, it is very versatile thanks to its compatibility with Ethernet, which allows the physical layer to use commercially available cables. It uses the "on the fly" principle for high-speed data exchange. As the frame passes, the slave nodes read and write data in the specific sector assigned to it in few nanoseconds. They have circular communication logic because all information arrives without distinction to the last slave and then returns to the sending master.

The protocol is standardized in IEC 61158 and is suitable for both hard and soft real-time requirements in automation technology. The goal during development of EtherCAT was to apply Ethernet for automation applications requiring short data update times (also called cycle times; $\leq 100 \mu \mathrm{s}$ ) with low communication jitter (for precise synchronization purposes; $\leq 1 \mu \mathrm{s}$ ) and reduced hardware costs. The transmission media that allows EtherCAT to operate is Ethernet protocol, based on the standard IEC 802.3. This standard supports several protocols always respecting the OSI model and, one of these, is EtherCAT. In what follows, the main features are described:

- Functional Principle: with EtherCAT, the Standard Ethernet packet or frame (according to IEEE 802.3) is no longer received, interpreted, and copied as process data at every node. The EtherCAT slave devices read the data addressed to them while the telegram passes through the device, processing data "on the fly". Similarly, input data are inserted while the telegram passes through. A frame is not completely received before being processed; instead 
Asian Journal of Basic Science \& Research Volume 2, Issue 4, Pages 59-73, October-December 2020

processing starts as soon as possible. Sending also is conducted with a minimum delay of small bit times. Typically, the entire network can be addressed with just one frame.

- Protocol: The EtherCAT protocol is optimized for process data and is transported directly within the standard IEEE 802.3 Ethernet frame using Ethertype 0x88a4.

- Performance: short cycle times can be achieved since the host microprocessors in the slave devices are not involved in the processing of the Ethernet packets to transfer the process images. All process data communication is handled in the slave controller hardware. Combined with the functional principle this makes EtherCAT a high performance distributed I/O system: Process data exchange with 1000 distributed digital I/O takes about $30 \mu$ s, which is typical for a transfer of 125byte over 100Mbit/s Ethernet. Data for and from 100 servo axis can be updated with up to $10 \mathrm{kHz}$. Typical network update rates are 1-30 kHz, but EtherCAT can be used with slower cycle times, too, if the DMA load is too high on your PC [16].

- Topology: EtherCAT enables a multitude of network topologies, including line, tree, ring, star, or any combination thereof. The protocol also enables a multitude of communication features such as cable redundancy, Hot Connect of segments, change of devices during operation, or even master redundancy with Hot Standby. Thus the combination of the topology variations and different network architectures enables numerous possibilities, e.g. sub-ordinated or neighboring control systems with consistent synchronization. Additional switches are not required. The physics of Ethernet allow a cable length of up to $100 \mathrm{~m}$ (300 ft) between two nodes. For higher distances, or the complete galvanic isolation between two slaves, fiber optic cables are used. With single-mode fiber, distances up to $20 \mathrm{~km}$ between two nodes can be bridged. Since a total of 65,535 nodes per network segment can be connected, the network extension is nearly unlimited.

- Synchronization: for synchronization a distributed clock mechanism is applied, which leads to very low jitter, significantly less than $1 \mu$ s, even if the communication cycle jitters, which is equivalent to the IEEE 1588 Precision Time Protocol standard (PTP). Therefore, EtherCAT does not require special hardware in the master device and can be implemented in software on any standard Ethernet MAC, even without dedicated communication coprocessor. To keep the clocks synchronized after initialization, the master or slave must regularly send out the broadcast again to counter any effects of speed difference between the internal clocks of each slave. The system clock is specified as a 64bit counter with a base unit of Ins starting at January 1, 2000, 0:00.

- Diagnosis: the fast, precise detection of disturbances is one of many diagnostic features of EtherCAT. Bit errors during transmission are detected reliably by the analysis of the CRC check sum: the 32 bit CRC polynomial has a minimum Hamming distance of 4. Besides the error detection and localization protocol, transmission physics and topology of the EtherCAT system allow an individual quality monitoring of every single transmission path. The automated analysis of the according error counters enables the exact localization of critical network segments.

- Device Profiles: the device profiles describe the application parameters and functional behavior of the devices, including device-specific state machines. Thus the migration to EtherCAT by adjusting the firmware and the hardware is simplified significantly. 
Referring to the Industry 4.0 introduced in the previous paragraph, EtherCAT fits perfectly with some of its principles. Since it is defined as a real-time field-bus, its communication system gets the "real time capability" principle. The overall system, thanks to the lowest allowable time cycle, can be controlled without significant delays. The flexibility of EtherCAT moreover defines the "modularity" principle that, with some minimum operations or settings, allows to immediately identify, in an already established plant, the eventual hardware changes based on EtherCAT system. However, their operability depends on the ability of the programmer, who knows the task and the pre-programmed applications very well. At last, the "Interoperability" principle is satisfied since the devices communicate with the same standard.

\section{Energy Evaluation through EtherCAT Variables}

In this paragraph, it will be shown how, based on the use of the EtherCAT protocol, it can be implemented an evaluation of the energy consumption related to an automated pick and place operation in an industrial plant. This procedure can be applied to one or more section of the industrial plant, therefore it is necessary to know in detail the functions, the parameters and time of execution of each activity. This can be done because all the plant variables are shared through the communication system in the EtherCAT environment and made available from any plant node.

\section{A. Energy consumption evaluation}

In the following the procedure is applied to the operation of a Cartesian robot operating in a Gantry type frame to perform pick-and-place operation. A more detailed description of the process referred to, including physical description and trajectories calculations is reported in [17]; for the purpose of the present paper a simplified description of the plant and movements refers to the schematics of Fig. 1.

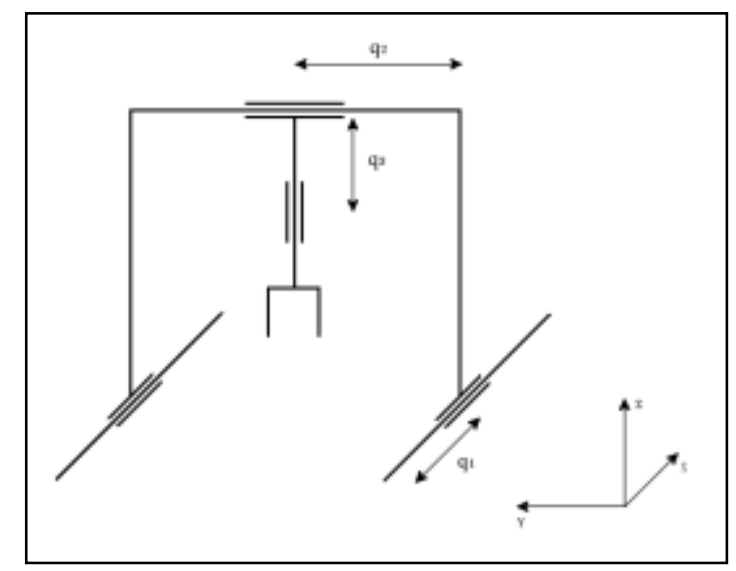

Figure 1: Schematics of the Pick-and-Place Gantry robot

When handling a product, the Pick and Place operation can be divided into three steps: i) lift on - is the vertical lifting; ii) travel - the transportation in horizontal direction; iii) set down - the release after a vertical drop. Using the Cartesian axes reference, the reference frame is placed along the Cartesian portal robot's so that the lift-on and set-down phases are accomplished along the z-axis. The travel phase is conducted along the $\mathrm{x}$ - and/or $\mathrm{y}$-axis in function of the final point position. The dynamic model of a manipulator provides a description of the relationships between the torque of the joints and the motion of the structure. In particular, the dynamic model comes from the matrix form of Euler-Lagrange equations: 
$\tau=D(q) \ddot{q}+C(q, \dot{q}) \dot{q}+G(q)$

Where, $\tau$ is the joint torque, $\mathrm{q}$ is the position of the reference point of the end-effector, $\mathrm{D}$ is the hybrid inertial matrix, $\mathrm{C}$ is the hybrid Coriolis matrix and $\mathrm{G}$ is the vector of gravity.

Let us consider the three prismatic joints with mass $\mathrm{m}_{11}, \mathrm{~m}_{12}$ and $\mathrm{m}_{13}$ actuated by identical motors with masses $\mathrm{m}_{\mathrm{m} 1}$, $\mathrm{m}_{\mathrm{m} 2}$ and $\mathrm{m}_{\mathrm{m} 3}$.

The velocity of the mass center for the joints is respectively:

$$
\begin{aligned}
& v_{c 1}=J_{v c 1} \dot{q} ; \quad v_{c 2}=J_{v c 2} \dot{q} ; \quad v_{c 3}=J_{v c 3} \dot{q} \\
& \text { with } \dot{q}=\left[\begin{array}{l}
\dot{q}_{1} \\
\dot{q}_{2} \\
\dot{q}_{3}
\end{array}\right] ; J_{v c 1}=\left[\begin{array}{lll}
1 & 0 & 0 \\
0 & 0 & 0 \\
0 & 0 & 0
\end{array}\right] ; \\
& J_{v c 2}=\left[\begin{array}{lll}
1 & 0 & 0 \\
0 & 1 & 0 \\
0 & 0 & 0
\end{array}\right] ; J_{v c 3}=\left[\begin{array}{ccc}
1 & 0 & 0 \\
0 & 1 & 0 \\
0 & 0 & -1
\end{array}\right]
\end{aligned}
$$

Hence the kinetic energy results:

$$
\begin{aligned}
E_{K}= & \frac{1}{2}\left(m_{1}+m_{2}+m_{3}\right) \dot{q}_{1}^{2}+\frac{1}{2}\left(m_{2}+m_{3}\right) \dot{q}_{2}^{2}+ \\
& +\frac{1}{2} m_{3} \dot{q}_{3}^{2}
\end{aligned}
$$

The inertial matrix, considering also the inertial components due to the motors rotation:

$$
\begin{aligned}
& D=\left[\begin{array}{ccc}
D_{11} & 0 & 0 \\
0 & D_{22} & 0 \\
0 & 0 & D_{33}
\end{array}\right] \\
& D_{11}=m_{l 1}+k_{r 1}{ }^{2} I_{m 1}+m_{l 2}+m_{m 2}+m_{l 3}+m_{m 3} \\
& D_{22}=m_{l 2}+k_{r 2}{ }^{2} I_{m 2}+m_{l 3}+m_{m 3} \\
& D_{33}=m_{l 3}+k_{r 3}{ }^{2} I_{m 3}
\end{aligned}
$$

Where, $\mathrm{k}_{\mathrm{ri}}$ is the transmission ratio of the motor $\mathrm{i}$ and $\mathrm{I}_{\mathrm{mi}}$ is the inertia moment around the motor axes. Since the joints are prismatic neither Coriolis nor centrifugal terms must be accounted for. Therefore, the matrix $\mathrm{C}$ is the null matrix. The potential energy holds only for the third joint:

$$
E_{p}=g\left(m_{1}+m_{2}+m_{3}\right) q_{3}
$$

Let the frictions assumed to be negligible. Hence, the dynamic equations are given by:

$$
\tau=\left[\begin{array}{ccc}
D_{11} & 0 & 0 \\
0 & D_{22} & 0 \\
0 & 0 & D_{33}
\end{array}\right] \ddot{q}+\left[\begin{array}{c}
0 \\
0 \\
g\left(m_{1}+m_{2}+m_{3}\right)
\end{array}\right]
$$


The actuators more commonly used to move the Cartesian robot here considered, and more in general for automation industry applications are the brushless motors, which in the following are modeled according to [18] with the model shown in Fig.2.

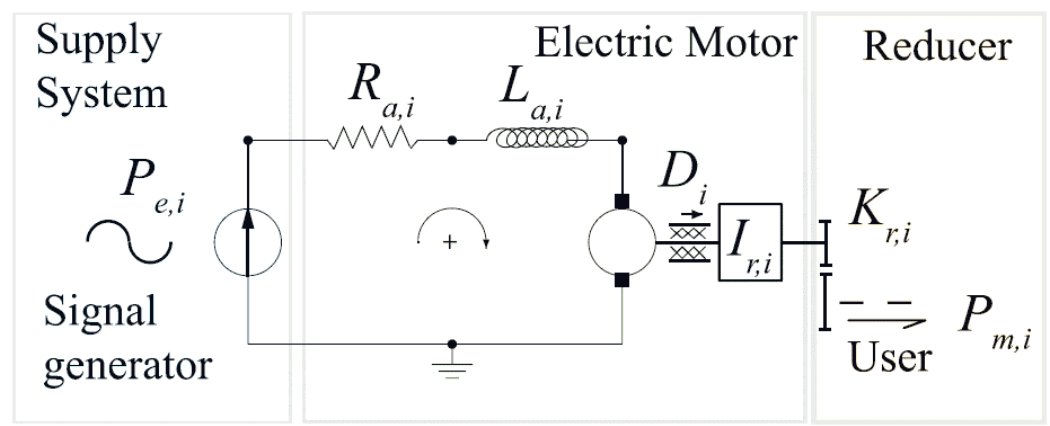

Figure 2: Electrical scheme of the robot actuators (Brushless motors)

Concerning the actuator and robot dynamics the total instantaneous electrical power Pe supplied to the manipulator is given by:

$$
P_{e}=\boldsymbol{i}_{a}^{T} \boldsymbol{v}_{\boldsymbol{a}}=\boldsymbol{i}_{\boldsymbol{a}}^{T} \boldsymbol{R}_{a} \boldsymbol{i}_{\boldsymbol{a}}+\boldsymbol{i}_{\boldsymbol{a}}^{T} K_{v} K_{r} \dot{q}_{a}
$$

Where: $\boldsymbol{v}_{\boldsymbol{a}}$ and $\boldsymbol{i}_{\boldsymbol{a}}$ are column vectors of the supplied armature voltages and currents respectively; $\boldsymbol{R}_{\boldsymbol{a}}, \boldsymbol{K}_{w}, \boldsymbol{K}_{r}$ are constant diagonal matrices of the different armature electric resistances, armature inductances, back EMF constants and gear ratios respectively.

According to [18] the mechanical losses can be neglected with respect to the electrical losses, thus the power consumption of the system can be reduced to the simple equation:

$$
P=\tau^{T} R_{1} \tau+\tau^{T} \dot{q}_{a}
$$

Where $\tau$ is the torque vector including the inertial effects due to the rotor inertias.

The overall energy necessary to perform a given operation for an industrial robot can be expressed as:

$$
E=E_{d}+E_{k m}+E_{g m}+E_{k l}+E_{g l}
$$

Where,

$\cdot E_{d}$ is the energy dissipated through the armature resistance and through the mechanical viscous friction

$\cdot E_{k m}$ is the kinetic energy stored in the manipulator inertial field

$\cdot E_{g m}$ is the gravitational potential energy due to the manipulator masses

- $E_{k l}$ is the kinetic energy delivered to the user

- $E_{g l}$ is the variation of the workpiece potential energy

The consumption of energy during pick and place operations however, considering a cycle time where the velocity of the handled object is picked up and delivered at zero velocity, is simplified by assuming: 
$E=E_{d}+E_{g l}$

The instantaneous power supplied to the manipulator is given by:

$P=\frac{\tau_{a v} \cdot v_{a v}}{60 \cdot 1000}[k W]$

Where, $\tau_{a v} \& v_{a v}$ are the torque actual value and the velocity actual value [rev/min]. Let $T_{w c}$ be the operation work cycle time that is the overall time programmed by the controller (e.g. PLC) to complete the pick-and-place operation. In this case the energy consumption for one PLC work-cycle, $E_{w c}$, can be calculated as:

$E_{w c}=\int_{0}^{T_{w c}} \frac{P}{360000} d t[k W h]$

The above described calculations can finally be implemented, even for different schemes and actuators, without further sensors and measurements, by exploiting the mechanical and electrical variables available on the system bus and within the PLC memory and normally used for control and diagnostics purposes. With the same assumptions even more complex models can be avoided.

\section{B. Using EtherCAT variables}

The above defined calculations are here executed by proposing a function block in EtherCAT standard, thus exploiting the variables already available in the process control with a direct procedure.

The EtherCAT modality to access to the bus is "Centralized" or "Master/Slave". The device Master - the network card EtherCAT - controls the bus and manages the accesses of Slave devices, according to Fig. 3 addressing table.

Figure 3: Master and devices address table

\begin{tabular}{l|l|l|l|l}
\hline Pos. $\Gamma$ & Address & Name (Type) & State requested & State actual \\
\hline$M$ & 0 & EtherCAT_Master & & \\
\hline 1 & 1002 & L_i700_SM & & \\
\hline 0 & 1001 & L_i700_SM_1 & & \\
\hline
\end{tabular}

The Master position is regulated by EtherCAT itself, while the Slave positions are controlled by the inverter, feeding and controlling the motors.

Each inverter has the drive motors as sub-slaves, as shown in Fig. 4.

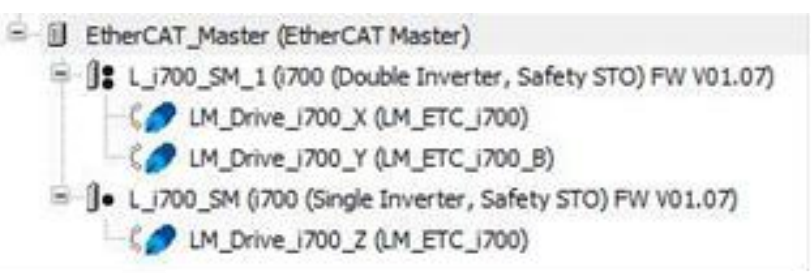

Figure 4: Sub-slave structure

In the following the operations are described with reference to the facilities by PLC Designer and DSD Drive Solution Design programs proposed in applications by Lenze [19]. Figure 5 screenshots is based on EhterCAT interfaces and macros and include the main variables involved for control and diagnostics of the drives. 
The cycle time is also a significant parameter to be properly defined in order to perform a reliable energy evaluation. It can be chosen by setting the cyclic interval down up to $1 \mathrm{~ms}$, which results a quite acceptable value for an energy detailed evaluation.

To test the EtherCAT function block capabilities the Energy consumption of the motor has been calculated by using the above mentioned variables, while the operation work cycle time $T_{w c}$ has been varied by using a scaling method suggested in [18]. At first a reference work cycle time $T_{w c}$ has been chosen; based on this criteria, the optimal trajectory parameters have been calculated. Then a number of different simulations have been executed, by assuming a scaled $\mathrm{T}_{\mathrm{wc}}$ work cycle, where value $<1$ implies that an accelerated process is considered, while value $>1$ is for slower processes. The trajectory parameters and the energy consumption calculated by the macros in EtherCAT are reported in Fig. 6 and Fig. 7, thus allowing a real time monitoring, to be further processed if required.

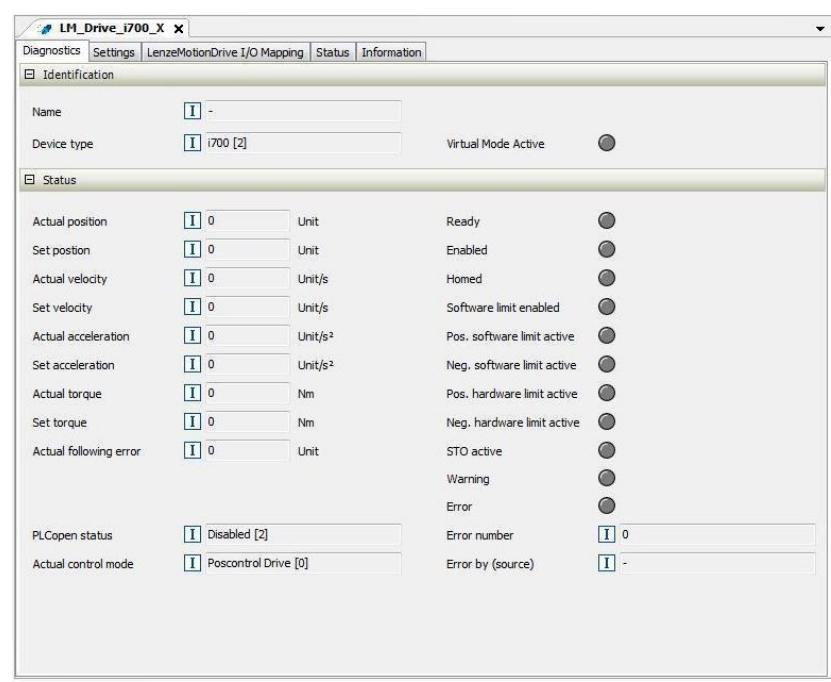

Figure 5: EtherCAT variables available, related to energy

\begin{tabular}{c|c|c|c|c|c|c|c|}
$\begin{array}{c}\text { SCALING } \\
\text { FACTOR }\end{array}$ & $\mathbf{0 , 5 4 3}$ & $\mathbf{1 , 0 0 0}$ & $\mathbf{1 , 2 2 8}$ & $\mathbf{1 , 4 5 5}$ & $\mathbf{1 , 6 8 3}$ & $\mathbf{1 , 9 1 0}$ & $\mathbf{2 , 1 3 8}$ \\
\hline$v_{z}[\mathrm{~m} / \mathrm{s}]$ & 0,230 & 0,125 & 0,102 & 0,086 & 0,074 & 0,065 & 0,058 \\
\cline { 2 - 8 }$v_{x}[\mathrm{~m} / \mathrm{s}]$ & 0,163 & 0,088 & 0,072 & 0,061 & 0,053 & 0,046 & 0,041 \\
\cline { 2 - 8 }$v_{y}[\mathrm{~m} / \mathrm{s}]$ & 0,163 & 0,088 & 0,072 & 0,061 & 0,053 & 0,046 & 0,041 \\
\cline { 2 - 7 }$a_{z}\left[\mathrm{~m} / \mathrm{s}^{2}\right]$ & 1,694 & 0,500 & 0,332 & 0,236 & 0,177 & 0,137 & 0,109 \\
$a_{x}\left[\mathrm{~m} / \mathrm{s}^{2}\right]$ & 1,198 & 0,354 & 0,235 & 0,167 & 0,125 & 0,097 & 0,077 \\
\hline$a_{y}\left[\mathrm{~m} / \mathrm{s}^{2}\right]$ & 1,198 & 0,354 & 0,235 & 0,167 & 0,125 & 0,097 & 0,077 \\
\cline { 2 - 7 }$T E T[\mathrm{~s}]$ & 30,49 & 56,13 & 68,90 & 81,68 & 94,45 & 107,23 & 120,00 \\
\hline
\end{tabular}

Figure 6: Energy related variables values vs Scaling factor

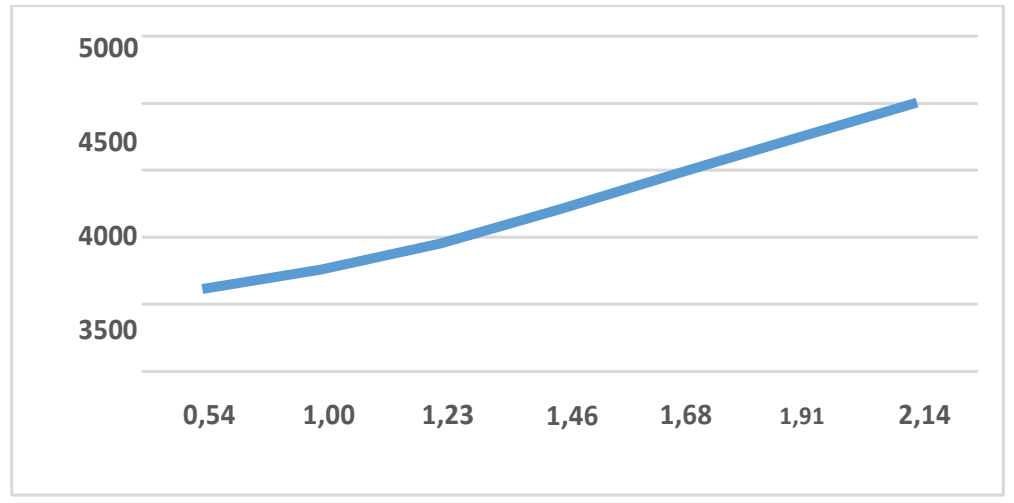

Figure 7: Energy vs Scaling factor 


\section{Sizing and Strategies of Coordination of an Automated Warehouse}

The second case study regards the operation performance improvement in an automated warehouse, available for improving to an Industry 4.0 profile. This type of plant is of increasing relevance due to the key role of logistics and distribution [20]. The plant considered in this study has an operative volume, with depth of 80 meters and a height of 16 meters; it has two runways, each occupied by a single-column, two degrees-of-freedom stacker crane [21]. The dynamic parameters of each machine (speed, position, voltage, currents) have been monitored, during normal operation, while travelling according to the trajectories used so far. Based on these data an energy model of the entire warehouse is reconstructed, by using similar equations as in the previous paragraph. The measurements were made by connecting the diagnostic cable, which was originally separated from the control network, to the control section of the actual plant, to be able to communicate with the supervising PLCs; the IP addresses of each inverter and drive are thus linked to the network, and therefore it is possible to monitor the field behavior. The ultimate goal is to be able to analyze and identify trajectory coordination strategies, so that not only the axes motion of a single stacker crane, but also the displacement of all the axes in the warehouse, be optimized for energy efficiency. In particular, it is desirable that all the stacker cranes be governed as to reduce the electrical energy absorption in the warehouse and to flatten the electrical power profile. The role of deterministic and real time data transmission throughout the plant is apparent, and the project is well coherent with Industry 4.0 principles (Interoperability, Real-Time capability). The energy model is implemented as before by using a software made available by Lenze for applications in industrial automation design (PLC Designer and DSD Drive Solution Design programs) [19]. After his validation based on the comparison with the measurement mentioned above, the same model will serve as a "Digital Twin", to test different trajectory solutions, satisfying to another of the Industry 4.0 principles, that is Virtualization.

From a comprehensive energy point of view, the solution closest to the technologies currently in use is to equip each machine drive with a regenerative module, capable of transmitting energy in bidirectional way to the network. In this way, it will be possible to recover energy during the braking, but also it will provide a physical means to transport and transfer energy among all the stacker cranes in the plant, whenever possible. As for the control strategies, a supervising processor at a higher level is required, capable of modifying the motion profiles of the stacker cranes in real time and as a function of the energy flow monitored in the network.

A dynamic trajectory planning can therefore be provided, as long as all the Cartesian coordinates are available; on the ther hand, an important piece of information, which can provide so much in terms of savings, is the mission time of the warehouse. Actually it happens in many warehouses that the mechanical parameters of the stacker crane are pushed up to the maximum acceptable value for performance, without taking into account the downtime of the machine to the storage bay, waiting for the new pallet.

Knowing precisely the number of missions/hours to complete, we can calculate the average time for each mission and set the mechanical parameters of the stacker crane, in order to avoid waste of energy, if this is not strictly required to make the warehouse more efficient. In fact, not always the maximum acceleration is required for all axes. Referring to the coordination methods, several proposals will be discussed. The results reported in the 
following deserve a preliminary discussion about the calculation of the actual value of power and energy involved during the operating cycles. As in the previous paragraph the power and the energy are derived from variables such as acceleration, speed and cycle time, so that we can trace the trends of the consumed energies and compare the different solutions. In the power evaluation, when it is evaluated in regular motor operation, flowing from the electrical source to the mechanical parts, the efficiency of both inverter and motor has been considered. When the energy is flowing from the mechanical side towards the DC bus condenser, during braking operation, the same efficiency parameters are accounted for in an inverse application.

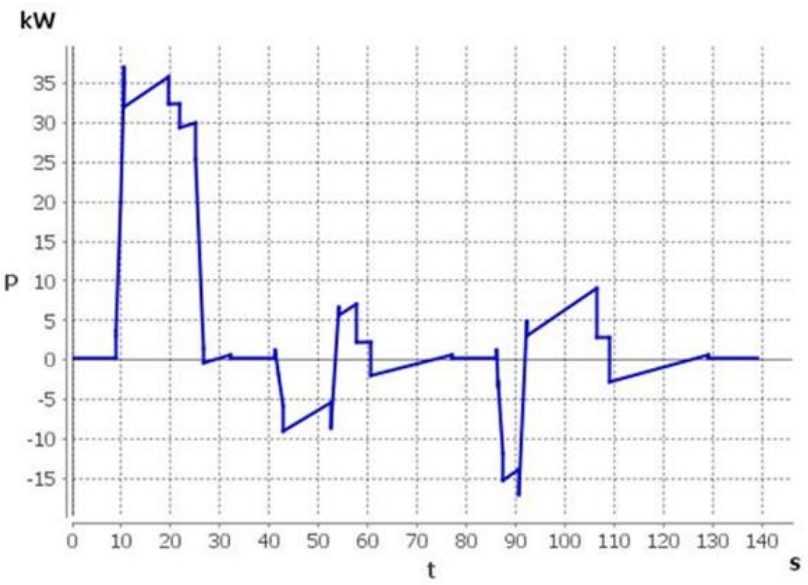

Figure 8: Absorbed electrical power in the worst condition (two stacker cranes operating at the same time, Solution A)

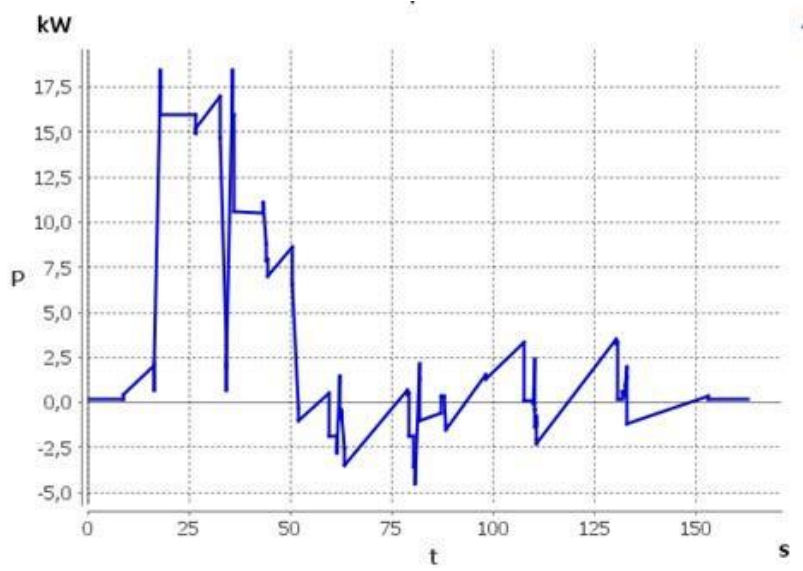

Figure 9: Absorbed electrical power for a delayed trajectory starting for the second crane (Solution B)

The energy consumption of the entire warehouse is first analyzed in the most critical situation, where both the stacker cranes perform their cycles at the same time (maximum power consumption, solution A). By assuming two same size stacker cranes, the absorbed power curve during a mission (one single displacement from Home position to Final destination) is reported in Fig. 8, showing a maximum of $37 \mathrm{~kW}$. An elementary coordination strategy, suggest to delaying the departures of the second stacker crane of a time equal to the sum of the acceleration and constant phase times (Solution B). In this case, with a similar energy consumption, the maximum power value 
drops by several $\mathrm{kW}$ (Figure 9), down to $18,5 \mathrm{~kW}$. This coordination strategy also guarantees a linear trend of the regenerated power curve.

On the other hand this strategy may lower the production efficiency of the warehouse, since the second stacker crane has to wait for the first to conclude its mission, by issuing more rigid criterion. From the measurement reported in the case study the warehouse cycle time is increased indeed from 140 to $160 \mathrm{~s}$. This leads to a $15 \%$ lowering of the yield. Therefore an economic balance is to be evaluated. In Fig. 10 they have been compared the two solutions from an economical stand point, by using a software tool of DSD, showing the reduction in Energy saving. Energy consumption in the first case has an economic value of $3383 € /$ year, while in the second case it is $2741 € /$ year. This allows a saving of $€ 642$ per year, a percentage of $19 \%$ of the initial cost. Therefore, energy efficiency gain exceeds the decrease in the production efficiency of the warehouse. On this basis many other solutions can be tested and evaluated, even dynamically, so that an optimum solution can be reached at the end [22].

\section{Conclusions}

The paper would like to demonstrate actual advantages deriving from the principles that identify the new industrial profile of I4. While the advantages range in a very large type of fields (from technical, to management, to economics) here the focus was on energy efficiency, where the more useful principles of I4 are interoperability and virtualization through a standard communication protocol (Industrial Ethernet).

Considerations about the relevant feature of an IE field bus EtherCAT have been made showing how it fits with I4. A first example, based on a Gantry robot, pointed out the ability of an I4 connected plant, to monitor the real time energy consumption, as a function of the work cycle. A second case, based on an actual warehouse, demonstrate the advantages coming from virtualization. An energetic model of the plant can be validated thanks to a number of variable reading and storage during actual operation, thus bringing to a reliable virtual plant image (Digital Twin).

Starting from that, a number of control strategies are simulated aimed at the optimization of some indexes, in our case the Energy Efficiency versus the plant performance, expressed as a number of missions/hour in the storing and retrieving operations. Further exploitation of the I4 features will be provided in an extension of this work, with reference to energy saving, by a more systematic analysis of trajectories coordination within the warehouse, even for a generalized number of trackway and stacker cranes.

\section{Acknowledgment}

The authors wish to acknowledge the relevant contribution of Lenze Automation Gmbh laboratories in Hameln and Milano to the experimental work referred to in the paper.

\footnotetext{
References

[1] Li Da Xu, Wu He, Shancang Li (2014), "Internet of Things in Industries: A Survey" - IEEE Transactions on Industrial Informatics, (Volume:10, Issue: 4 ) RFID Virtual Journal, IEEE.

[2] Ioan Ungurean, Nicoleta-Cristina Gaitan and Vasile Gheorghita Gaitan, “An IoT architecture for things from industrial environment", 10th International Conference on,Communications (COMM), 2014.
} 
Asian Journal of Basic Science \& Research Volume 2, Issue 4, Pages 59-73, October-December 2020

[3] H Madsen, G Albeanu, B Burtschy et al., "Reliability in the Utility Computing Era: Towards Reliable Fog Computing”, 20th International Conference on Systems, Signals and Image Processing (IWSSIP), 2013.

[4] Zhiqiang $\mathrm{Xu}$, Jialiang He, Zhiyong Chen, "Design and Actualization of IoT-based Intelligent Logistics System", IEEE International Conference on Industrial Engineering and Engineering Management (IEEM), 2012.

[5] Jie Yin, Jun Li, Peichao Ke, “A Provenance Based Scheduling Algorithm for Logistics Chain in IOT”, 6th Int. Conf. on Info. Management, Innovation Management and Industrial Engineering (ICIII), 2013 (Volume:1).

[6] Min-Hsiung Hung, Yu-Chuan Lin, Tran Quoc Huy, Haw-Ching Yang, et al. , "Development of a cloud-computing-based equipment monitoring system for machine tool industry", IEEE International Conference on Automation Science and Engineering (CASE), 2012.

[7] Maria Rita Palattella, Nicola Accettura, Luigi Alfredo Grieco, Gennaro Boggia, Mischa Dohler, and Thomas Engel, "On Optimal Scheduling in Duty-Cycled Industrial IoT Applications Using IEEE802.15.4e TSCH, Sensors Journal, IEEE (Volume:13, Issue: 10), 2013.

[8] C.K.M. Lee, Yaqiong Lv, K.K.H. Ng, William Ho \& K.L. Choy (2017) Design and application of Internet of things-based warehouse management system for smart logistics, International Journal of Production Research, DOI: 10.1080/00207543.2017.1394592.

[9] Ding W. (2013) Study of Smart Warehouse Management System Based on the IOT. In: Du Z. (eds) Intelligence Computation and Evolutionary Computation. Advances in Intelligent Systems and Computing, vol 180. Springer, Berlin, Heidelberg.

[10] LI Zhong-Cheng (2011) Design and Implementation of the Internet of Things in Intelligent Warehouse Management, Computer Systems \& Applications, 2011-07.

[11] Qu, T., Lei, S.P., Wang, Z.Z. et al., IoT-based real-time production logistics synchronization system under smart cloud manufacturing, Int J Adv Manuf Technol (2016) 84: 147.

[12] ACATECH (National Academy of Science and Engineering-Germany), "Recommendations for implementing the strategic initiative INDUSTRIE 4.0. Final report of the Industrie 4.0 Working Group" -

Available on

https://en.acatech.de/publication/recommendations-for-implementing-the-strategic-initiative-industrie-4-0-final-r eport-of-the-industrie-4-0-working-group/download-pdf?lang=en

[13] M Hermann, T Pentek, B Otto: “Design Principles for Industrie 4.0 Scenarios: A Literature Review”, Technische Universität Dortmund, 2015-Available on:

http://www.snom.mb.tu-dortmund.de/cms/de/forschung/Arbeitsberichte/Design-Principles-for-Industrie-4_0-Sce narios.pdf

[14] P. S. Marshall, J. S. Rinaldi "How to Plan, Install and Maintain TCP/IP Ethernet Networks", ISA, 2004 ISBN 1-55617-869-7 pp. $1-4$. 
[15] M. Felser: "Real-Time Ethernet-Industry Prospective" - Proceedings of the IEEE, vol. 93, no. 6, pp. 1118-1129, June 2005, doi: 10.1109/JPROC.2005.849720.

[16] Gunnar Prytz: “A Performance Analysis of EtherCAT and Profibus IRT”, Proc. Of 13th IEEE International Conference on Emerging Technologies and Factory Automation (ETFA), Hamburg, September 2008.

[17] S. Farné, E. Bassi, F. Benzi and F. Compagnoni, "IloT based efficiency monitoring of a Gantry robot," 2016 IEEE 14th International Conference on Industrial Informatics (INDIN), Poitiers, 2016, pp. 714-719, doi: 10.1109/INDIN.2016.7819252.

[18] M Pellicciari, G Berselli, F Leali, A Vergnano (2013). A method for reducing the energy consumption of pick-and-place industrial robots. Mechatronics, 2013 - Elsevier.

[19] Drive Solution Designer V4.3 (Lenze). Available at:

http://www.lenze.com/en/application-knowledge-base/article/201103523/1/University Science, 1989.

[20] K. J. Roodbergen, I. F. A. Vis, “A survey of literature on automated storage and retrieval systems”, European Journal of Operational Research 194 (2009) 343-362, Available online at www.sciencedirect.com

[21] F. Benzi, E. Bassi, F. Marabelli, N. Belloni and M. Lombardi, "IIoT-based Motion Control Efficiency in Automated Warehouses," 2019 AEIT International Annual Conference (AEIT), Florence, Italy, 2019, pp. 1-6, doi: 10.23919/AEIT.2019.8893370.

[22] J.Tavčar, I. Horváth, “A Review of the Principles of Designing Smart Cyber-Physical Systems for Run-Time Adaptation: Learned Lessons and Open Issues”, IEEE Transactions on Systems, Man, and Cybernetics: Systems Year: 2018, ( Early Access ) Pages: $1-14$. 